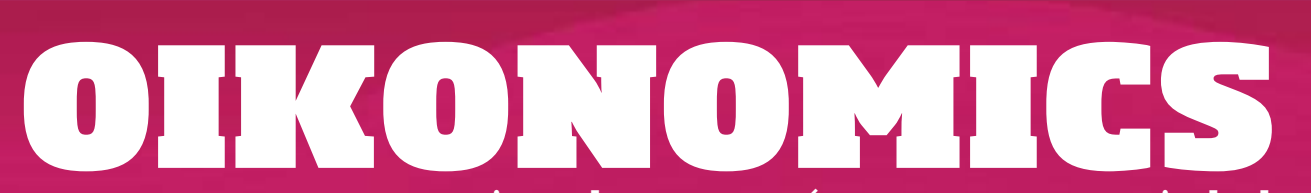

Revista de economía, empresa y sociedad

INTELIGENCIA ARTIFICIAL

\title{
Fl empleo ante la nueva oleada digital: ¿robots humanos 0 recursos humanos?
}

\section{Joan Torrent-Sellens}

Profesor de los Estudios de Economía y Empresa (UOC)

RESUMEN La preocupación por el futuro del trabajo es un tema recurrente cada vez que se evidencia un proceso de cambio disruptivo en la tecnología. El análisis económico ha señalado que la tecnología no destruye el trabajo, sino que sesga habilidades y destrezas, y desplaza tareas, empleos, ocupaciones y personas. En general, a largo plazo, las consecuencias de estas oleadas tecnológicas sobre el trabajo suelen ser positivas, porque se vinculan con incrementos de la productividad, nueva actividad económica, más ocupación y mejoras salariales para las personas que trabajan en las empresas o los sectores vinculados con la innovación tecnológica. Además, estos efectos positivos suelen compensar en el largo plazo los efectos de sustitución del empleo si las empresas, especialmente a través de sus políticas de recursos humanos, actúan en forma de políticas activas, que formen y recapaciten a las personas desplazadas. Esta forma general de interacción de la tecnología con el trabajo se ha puesto en entredicho con la reciente oleada digital, caracterizada, entre otras cosas, por la explosión de la robótica inteligente. Según algunos autores, el ritmo de sustitución del trabajo humano por parte de los robots será tan elevado que difícilmente se podrán compensar por la vía habitual de incrementos de demanda y productividad. Otros autores defienden justo lo contrario y enmarcan la dinámica actual en el contexto de las interacciones tradicionales entre tecnología y trabajo. Pero la robótica es trabajo no humano, tiene unas características muy particulares y dinámicas, ofrece un amplio abanico de posibilidades de utilización y, al mismo tiempo, también genera miedos. En este artículo, analizaremos las 
implicaciones para el empleo de la nueva robótica, con especial atención a las repercusiones para la dirección y la gestión de recursos humanos.

PALABRAS CLAVE robótica; inteligencia artificial; empleo; desigualdad; gestión de recursos humanos

\section{Employment in the new digital wave: human robots or human resources?}

ABSTRACT Concern for the future of employment is a recurring theme whenever a process of disruptive change in technology takes place. Economic analysis has shown that technology does not destroy work, but it skews skills and abilities, and displaces tasks, jobs, occupations and people. Generally, in the long term, the consequences of these technological waves on work tend to be positive because they are linked to increases in productivity, new economic activity, higher employment and salary improvements for people working in firms or sectors related to technological innovation. In addition, the effects of job substitution can be offset in the long term if firms' strategies and policies, especially in terms of human resource management, take the form of active employment policies that train and reskill displaced people. This general form of interaction of technology with work has been questioned with the recent digital wave characterized, among other factors, by the explosion of intelligent robotics. According to some authors, the rate of substitution of human labour by robots will be so fast that they can hardly be compensated by the usual route of increases in demand and productivity. Other authors argue just the opposite, and frame the current dynamics within the context of the traditional interactions between technology and work. However, robotics is non-human work, has very particular and dynamic characteristics, offers a wide range of possibilities of use and, at the same time, generates fears too. In this article, we will analyse the employment implications of new robotics, paying special attention to the human resources management.

KEYWORDS robotics; artificial intelligence (Al); employment; inequality; human resource management (HRM) 


\section{Introducción}

La consolidación de la economía global del conocimiento y la progresiva articulación de la empresa y los negocios en red han venido transformando muy profundamente, de manera estructural, el empleo. Poco a poco, el trabajo homogéneo y manual, la organización industrial del empleo (atomización, jerarquía y falta de autonomía en el puesto de trabajo), el trabajo estable para toda la vida en una misma empresa, los períodos separados de formación y empleo, la retribución únicamente fija y un marco de relaciones laborales, con un contrato social que intercambia horas homogéneas de trabajo y salarios fijos por productividad, se van agotando lentamente. En sustitución de las formas tradicionales de empleo, se consolida una nueva forma de trabajo en red. Se trata de la forma típica, en el sentido de fundamental, de estructurar el trabajo ante las nuevas condiciones de innovación, flexibilidad y bienestar que demanda la economía global del conocimiento. El trabajo en red, que amplifica el trabajo cognitivo no rutinario y sustituye al trabajo no cognitivo y cognitivo rutinario, se caracteriza por cinco elementos clave (Torrent-Sellens y Ficapal-Cusí, 2009):

- En primer lugar, por la intensidad de las competencias en el uso de las tecnologías de la información y la comunicación (TIC), o competencias digitales.

- En segundo lugar, por una estrategia competitiva de las empresas basada en la innovación como instrumento imprescindible para competir flexiblemente en la economía global del conocimiento.

- En tercer lugar, por una organización del trabajo flexible, lejos de los esquemas tayloristas y fordistas, con un trabajo autónomo por equipos y proyectos, una toma de decisiones autónoma y descentralizada y una supervisión basada en objetivos/resultados.

- En cuarto lugar, por unos trabajadores cualificados, polivalentes, comprometidos y con una división del trabajo basada en el conocimiento de todo tipo, no únicamente el científico y tecnológico.

- Y, en quinto lugar, por unas prácticas de gestión de los recursos humanos avanzadas, con unos mecanismos de selección y trabajo por competencias, formación y cualificación constante, formas de retribución variable y un marco de relaciones laborales flexible pero estable. En este contexto, aparece un nuevo contrato social que intercambia conocimiento, innovación, trabajo heterogéneo y compromiso por retribuciones, formales e informales, basadas en el cumplimiento de objetivos y con la incorporación de los trabajadores en la toma de decisiones. Este nuevo contrato social intercambia competitividad por gestión colaborativa. 
Pero sobre este proceso de consolidación del trabajo en red, durante los últimos años se está construyendo un nuevo escenario de cambio para el mundo laboral. Sobre las transformaciones vinculadas con la tercera revolución industrial, la de las TIC y de internet, se ha generado una nueva oleada de cambio tecnológico disruptivo, que algunos, quizá precipitadamente, ya han denominado cuarta revolución industrial, pero que empieza a generar también importantes transformaciones sobre los mercados de trabajo. Todo parece indicar que estamos a las puertas de una nueva oleada tecnológica de utilidad general, que refuerza y profundiza la oleada de las tecnologías digitales y la era de la información y el conocimiento (tercera revolución industrial). La robótica, la inteligencia artificial, el aprendizaje de las máquinas o aprendizaje profundo, la computación en la nube, los grandes datos, la impresión 3D, internet de la cosas, la nanotecnología y la biotecnología parece que se configurarán como una base tecnológica interconectada de un nuevo paradigma técnico-económico que se interrelacionará con cambios sociales y culturales de primera magnitud (Torrent-Sellens, 2015). Esta nueva oleada tecnológica, que se materializará con fuerza durante los próximos años, tiene implicaciones fundamentales en la explicación de la productividad y la estructura del empleo. A lo largo del artículo, revisaremos estas implicaciones y pondremos especial énfasis en los efectos sobre el trabajo de la robótica inteligente y sus implicaciones para las prácticas de recursos humanos. Para contestar a esta pregunta, lo primero que debemos establecer es ¿cómo está evolucionando la robótica y en qué dirección?

\section{Revolución cámbrica en la robótica}

Según la Federación Internacional de Robótica (IFR, 2017), un robot es un manipulador multipropósito, controlado automáticamente, reprogramable y que puede ser fijo o móvil. Durante los últimos años, los datos disponibles nos señalan una presencia claramente creciente y una amplia diversificación de utilidades de los robots industriales (utilizados en los procesos de automatización industrial). Según las previsiones de la IFR, en 2020 más de 2,5 millones de robots serán utilizados por la industria mundial, a un ritmo de crecimiento anual que superará el 10 \% y con un número de unidades que duplicará sobradamente el de los robots que había a principios de la década (poco más de un millón en 2010). A pesar de que más del $70 \%$ de los robots instalados en la actualidad se ubican principalmente en cuatro ramas de actividad industrial -automoción, electricidad y electrónica, metalurgia y química-, durante los próximos años se prevé una expansión hacia otras ramas, como resultado de cuatro tendencias detectadas: 
- En primer lugar, gracias a los avances en la compactación y usabilidad de los robots que determina la introducción de las nuevas aplicaciones en inteligencia artificial y programación.

- En segundo lugar, por la creciente colaboración entre humanos y robots, lo que permite la complementariedad de tareas y un trabajo conjunto mucho más eficiente que si se realiza por separado.

- En tercer lugar, la incorporación de las PYMES al mundo de la robótica, lo que permitirá un crecimiento muy amplio de su base de aplicación.

- Y, en cuarto lugar, las mejoras de eficiencia económica y ambiental que la robótica introduce y que la actividad industrial debería adoptar para desarrollar un sistema productivo más limpio y sostenible, además de eficiente.

La mayoría de investigadores coincide en que, en la actualidad, la robótica experimenta una explosión vinculada con un crecimiento exponencial de su diversificación y aplicabilidad. Algunos denominan a este período revolución cámbrica, en el sentido de que la robótica vive una situación similar a la que vivió la vida en la tierra hace unos 500 millones de años (Pratt, 2015). Esta revolución estaría vinculada con el hecho de que muchas de las tecnologías de base que se aplican sobre la robótica, en especial la informática (rendimiento informático; instrumentos de diseño electromecánico y fabricación controlada numéricamente), el almacenaje de energía y datos (poder computación global) y las comunicaciones (inalámbricas e internet), han crecido exponencialmente en potencia y capacidad durante los últimos años. Además, dos nuevas tecnologías, la robótica en la nube (cloud robotics) y el aprendizaje en profundidad (deep learning) podrían retroalimentarse del crecimiento de las tecnologías básicas y generar un círculo virtuoso de diversificación y aplicabilidad de la robótica durante los próximos años. La robótica en la nube permite a los robots aprender de la experiencia de otros robots, en especial a medida que crece su número. Los algoritmos de aprendizaje profundo son métodos pensados para que los robots aprendan y generalicen su aprendizaje sobre la base de conjuntos de adiestramientos muy grandes (grandes datos en la nube). Esta nueva fase de la robótica se sustenta en cuatro fundamentos principales:

- En primer lugar, la autonomía basada en la memoria. Los avances en el rendimiento de la computación y la capacidad de almacenaje de datos han permitido a los investigadores de la robótica explorar nuevos métodos basados en la memoria para resolver los tradicionales problemas de percepción, planificación y control que limitaban la autonomía de los robots. Gracias a los algoritmos de búsqueda rápida en internet y a la computación en la nube, hoy los robots pueden acceder a un gran número de experiencias previas que pueden ser utilizadas para guiar una respuesta. Si no hay memoria anterior coincidente, el robot 
puede pedir ayuda a las personas. Una vez ha recibido la ayuda, la respuesta se codifica y ya puede ser utilizada en forma de memoria por otros robots.

- En segundo lugar, la posibilidad de compartir experiencias a alta velocidad. A diferencia de las personas, por el momento imbatibles en su velocidad de comunicación interna (redes neuronales de su cerebro), los robots son claramente superiores en su capacidad de comunicación externa. Una combinación de comunicación inalámbrica a través de internet puede ser utilizada para que los robots compartan lo que van aprendiendo. Mientras que las personas tardan mucho más tiempo en aprender cosas suficientes para agregar de manera significativa a su compendio de conocimiento común, los robots pueden hacerlo mucho más rápida y eficientemente a través de la velocidad de comunicación externa.

- En tercer lugar, la posibilidad de aprender a través de la imaginación. Las personas utilizan la imaginación para practicar o prepararse para afrontar circunstancias futuras. Del mismo modo, un robot que usa sus capacidades de computación en la nube puede usar la simulación para explorar futuras circunstancias y experimentar soluciones. Rápidamente las soluciones exitosas pasarán a formar parte del bagaje de conocimiento acumulado de la robótica en la nube, lo que mejora el rendimiento de todos los robots.

- Y, en cuarto lugar, la posibilidad de aprender de las personas. La percepción sigue siendo uno de los retos más importantes que la robótica debe afrontar para la mejora de la autonomía de los robots. A través de las recientes mejoras en la capacidad para el tratamiento y el análisis de grandes conjuntos de datos, la robótica puede avanzar muchísimo en este sentido. A título de ilustración, todo el conjunto de contenidos digitales que las personas compartimos en los medios de comunicación y redes sociales son un recurso enorme que, con la suficiente capacidad de computación, los robots utilizarán, por ejemplo para identificar, asimilar o verificar comportamientos.

Pero ¿cuáles son las implicaciones para la economía y el trabajo que puede tener esta revolución cámbrica que se espera para la robótica inteligente durante los próximos años? A continuación, las analizaremos.

\section{Robótica y empleo: ¿complementariedad o sustitución?}

Parece evidente que esta nueva generación de robots inteligentes podría contribuir a generar más valor y riqueza a través de nueva actividad, aumentos de productividad, nuevo empleo, y más salarios y demanda. En este contexto, durante los últimos años, la investigación ha consolidado una visión de efectos optimista, que 
entronca con la teoría clásica sobre el cambio tecnológico sesgador de habilidades y su posibilidad de crear puestos de trabajo y recolocar a los empleados excluidos a través de la formación, el aprendizaje y la política pública (Autor, 2015). Esta visión optimista esgrime un conjunto de tres argumentos básicos:

- En primer lugar, a pesar de que los ordenadores y los robots han asumido tareas vinculadas con el trabajo de oficina y los trabajos rutinarios, al mismo tiempo también han proliferado puestos de trabajo que requieren importantes dosis de creatividad y habilidades para la resolución de problemas. Estos nuevos puestos de trabajo vinculados con la innovación, en muchas ocasiones apoyados con tareas de computación y comunicación realizada por ordenadores e Internet, crecen en todo el mundo.

- En segundo lugar, en paralelo con el crecimiento de los puestos de trabajo de elevada cualificación, los empleos de baja cualificación también han aumentado para aquel conjunto de tareas que son difíciles de automatizar, como algunas de las tareas que realizan los empleados de la restauración, conserjes o tareas del hogar.

- Y, en tercer lugar, en general los resultados de la primera oleada tecnológica digital, la de las TIC e internet, han sido favorables sobre el empleo. La investigación sobre el cambio tecnológico digital sesgador de habilidades ha demostrado que el uso de ordenadores e internet se ha asociado con niveles más elevados de ocupación y salarios para los trabajadores con las competencias digitales y educativas necesarias. Sin embargo, como toda oleada de innovación tecnológica, la postura más optimista también reconoce que la robotización y la digitalización cambiarán profundamente los mercados de trabajo, en el sentido de que pueden generar grandes eficiencias que reduzcan los tiempos de trabajo. Ahora bien, esta liberación de tiempo, que requeriría la reestructuración y reeducación de muchos trabajadores, también podría acabar generando nuevos nichos de ocupación, por ejemplo en la industria del ocio, viajes y turismo. En todo caso, el flujo económico podría continuar generando nuevas oportunidades de empleo basadas en la creatividad y la innovación. Inclusive, es posible plantear escenarios de trabajo alternativo, contingente, donde buena parte de la generación de rentas provenga de la robótica y los trabajadores humanos puedan plantearse otros tipos de contribución económica y social.

Sin embargo, durante los últimos años también ha proliferado una aproximación más pesimista. Esta defiende que la nueva oleada de innovaciones tecnológicas digitales estaría destruyendo masivamente puestos de trabajo. Esta postura, contrastada principalmente para los mercados de trabajo en Estados Unidos, destaca diversos argumentos (Brynjolfsson y McAfee, 2012; 2014): 
- En primer lugar, que la recesión económica en Estados Unidos durante el período 2007-2010 consolidó pérdidas permanentes de puestos de trabajo. Es decir, que una vez superada la crisis y llegada la recuperación, la creación de puestos de trabajo no se aceleró. Este fenómeno se ha reconocido como recuperación sin creación de empleo (jobless recovery).

- En segundo lugar, se constata también una creciente divergencia entre los incrementos de productividad y los aumentos de ocupación en Estados Unidos desde el año 2000. A diferencia de lo sucedido en otros ciclos económicos, estas dos magnitudes no evolucionan en concordancia (la productividad crece y la ocupación se mantiene estable), de manera que las mejoras de eficiencia no se trasladan al mercado de trabajo. La tecnología digital sería la principal responsable de este desencaje.

- Y, en tercer lugar, la disminución de la ocupación ya no se limitaría solo a los puestos de trabajo de la industria deslocalizada en México o China. Los trabajadores de cuello blanco, y los profesionales y técnicos cualificados, ya no serían inmunes a las pérdidas permanentes de puestos de trabajo. Como consecuencia, los niveles medios de ingreso y renta disponible de los trabajadores no crecerían, a pesar de los aumentos del producto generado. Todo ello acabaría redundando en notables aumentos de la desigualdad, porque las rentas generadas por los avances en el ingreso se quedan en pocas manos. En perspectiva histórica se trataría de una gran paradoja: innovación y productividad en niveles récord y, al mismo tiempo, caídas de ingresos, menos puestos de trabajo y aumento de la desigualdad. Esta paradoja estaría relacionada con el hecho de que la nueva oleada tecnológica digital avanzaría tan rápido y abarcaría un conjunto tan amplio de nuevas aplicaciones que las habilidades de las personas y las organizaciones no podrían seguir su ritmo (Rotman, 2013).

A pesar de que la aproximación pesimista defiende la utilidad de las oleadas tecnológicas anteriores, que habrían mejorado ostensiblemente la ocupación, salarios, riqueza y calidad de vida en todo el mundo, en el caso de la oleada actual, se defiende justo lo contrario. Este argumento pesimista se asienta sobre un conjunto de premisas (Ford, 2015):

- En primer lugar, sobre la predicción de que alrededor de la mitad de los puestos de trabajo actuales podrían ser automatizados, aunque parcialmente (Frey y Osborne, 2013).

- En segundo lugar, las tendencias actuales en los mercados de trabajo, en especial los aumentos de la dispersión salarial, la disminución de la participación del trabajo en la renta nacional y los aumentos del paro estarían vinculados con la automatización y la digitalización. 
- En tercer lugar, la caída de precios de los ordenadores y el aumento de capacidad de los procesadores harían más atractiva la sustitución de trabajadores por robots.

- Y, en cuarto lugar, el actual progreso en robótica e inteligencia artificial dirigirían hacia una nueva dirección las tradicionales relaciones entre innovación tecnológica y trabajo. Los avances en tecnología robótica permitirían la creación de robots inteligentes, fáciles de programar y con capacidades para realizar un trabajo cognitivo que sustituiría masivamente el trabajo humano. En este contexto de sustitución masiva del empleo, para solventar los problemas sobre los mercados de trabajo de la oleada tecnológica vinculada con la robótica inteligente, se haría del todo necesaria una reestructuración en profundidad de todo el sistema económico. Las medidas compensatorias tradicionales, como las prestaciones de paro o las pensiones, inclusive la educación o la actualización de habilidades, resultarían insuficientes para resolver los problemas de los trabajadores desplazados.

En resumen, esta aproximación más pesimista sustenta que la velocidad de sustitución de habilidades humanas por parte de los robots puede generar situaciones de exclusión muy importantes, puesto que el tiempo de adaptación demandado a la fuerza de trabajo será mucho más rápido y la oferta de capacidades (cuerpos que hacen trabajo manual y mentes que hacen trabajo cognitivo) de las personas se irá reduciendo. De hecho, se postula que el aumento de las capacidades de los robots reducirá aceleradamente el valor del trabajo humano en muchos sectores de actividad, hasta el punto de que no habrá tiempo de recuperar el trabajo desplazado a través de la generación de nuevos empleos en nuevas empresas. En este contexto, se postula un futuro donde muchos de los bienes materiales que la mayoría de la gente demanda pueden ser producidos a bajo coste por robots. Esta economía podría evolucionar de varias maneras. Pero los problemas de desigualdad serían evidentes si pocas personas con talentos excepcionales y no replicables por parte de los robots recibieran ingresos muy elevados, mientras que la mayoría de personas tendrían que contentarse con unos niveles de ingresos muy bajos.

\section{Conclusión: recursos humanos y robots. ¿Es posible la co-robótica?}

Acabamos de constatar que se va acumulando evidencia acerca de los efectos positivos de la robótica inteligente sobre la productividad y con efectos mixtos sobre el empleo. Pero, más allá de los cambios en la composición de la fuerza de trabajo hacia una creciente polarización del empleo en ocupaciones cognitivas de eleva- 
dos ingresos y ocupaciones manuales de bajos ingresos, con un amplio espectro de crecimiento para la tecnología en los empleos rutinarios y de ingresos medios, el efecto de la nueva oleada tecnológica sobre el futuro del empleo está lejos de la certidumbre (Bessen, 2016).

Es verdad que la automatización, la digitalización y la robótica se expandirán a través de la sustitución del empleo rutinario y que este proceso es claramente ahorrador de empleo (Frey y Osborne, 2013). Ahora bien, no existe evidencia que demuestre que la revolución digital, la primera oleada de innovaciones tecnológicas disruptivas vinculadas con las $\mathrm{TIC}$ e internet, haya reducido la demanda global de empleo, más bien todo lo contrario. Inclusive, los sectores tecnológicamente menos avanzados de la economía, como algunos sectores públicos, o algunas dimensiones empresariales menos proclives a la digitalización, como las PYMES, siguen creando empleos a partir de la revolución digital. A medida que la automatización y la robótica se expandan hacia los sectores más tecnológicamente atrasados, la innovación y la productividad deberían generar incrementos de renta en forma de mayores salarios o mayor capacidad de compra de otros productos. Así pues, más allá de un desfase mayor que en el pasado entre ocupación perdida y ocupación generada en el corto plazo, nada sugiere que, en el largo plazo, la nueva oleada digital de la automatización robótica y la inteligencia artificial no sea capaz de explotar los beneficios para el empleo que ya ha aprovechado otras oleadas tecnológicas anteriores.

De hecho, muchas de las previsiones sobre la sustitución del trabajo a raíz de la nueva oleada de la digitalización no tienen presente la posibilidad de un cambio en la relación de habilidades entre personas y máquinas, lo que podría redundar en aumentos de eficiencia y reforzar las relaciones de complementariedad por encima de las relaciones de sustitución. En este sentido, la co-robótica, es decir la posibilidad de que los robots y las personas trabajen juntos y mejoren conjuntamente la eficiencia de su trabajo (por encima de las tareas realizadas por separado), ya empieza a ser una realidad. Por otra parte, la investigación también ha puesto de relieve la importancia del nivel general de demanda (Autor, 2015). A pesar de la sustitución de tareas generada por la tecnología, los vínculos entre las personas en las tareas no sustituidas tienden a reforzarse. La nueva oleada de la robótica inteligente tiende a eliminar empleos (puestos de trabajo, ocupaciones o tareas), pero no el trabajo. El cambio tecnológico, junto con las otras formas del cambio económico, es un determinante importante de los puestos de trabajo, las industrias y las personas afectadas por el paro y la descualificación. Pero el nivel general de demanda de bienes y servicios es el factor más importante que determina cuántas personas se ven afectadas, por cuánto tiempo estarán en el paro y lo difícil que es para los nuevos integrantes del mercado de trabajo encontrar un empleo. 
En esta aproximación dinámica, la relación entre la nueva oleada digital, la robótica y el empleo también se matizaría en el sentido de que en la economía habría fuerzas de autocorrección que compensarían los efectos negativos identificados (Acemoglu y Restrepo, 2015). En especial, se ha señalado que el proceso innovador vinculado con la automatización y el trabajo más cognitivo podrían ir en paralelo, y que la inversión en robótica reduciría la relación entre el salario y la tasa de rendimiento de las empresas, lo que podría hacer más atractiva la inversión en trabajo humano cognitivo. Del mismo modo, estas fuerzas autocorrectoras (compensación en la inversión entre trabajo cognitivo y no cognitivo) se podrían también en marcha para compensar algunas de las desigualdades generadas entre los trabajadores con distintas habilidades.

Pero más allá de los modelos, investigaciones y predicciones, la investigación pone de relieve que la dinámica de los mercados de trabajo es muy compleja y obedece a un amplio conjunto de fuerzas impulsoras y detractoras. A pesar de las diferencias de visión detectadas sobre las tareas, empleos y actividades económicas que se verán más afectadas por la nueva oleada digital de la robótica inteligente, existe un claro consenso que señala que la fuerza de trabajo -actual y futura- debería dotarse de las suficientes habilidades y cualificaciones para que las personas puedan aprovechar las mejores condiciones de trabajo y salarios que impulsan la automatización, la computarización, la robótica y la inteligencia artificial. Esta tarea de equiparar las generaciones actuales y futuras de trabajadores con las habilidades necesarias para trabajar con los robots, y con todo tipo de maquinaria y aplicaciones digitales de nueva generación, recae tanto en las personas como en las empresas.

Sin duda, el reto que plantea la nueva oleada digital y la robótica inteligente es mayúsculo, tanto para las personas como para las empresas. Las posibilidades de que las empresas se suban a esta nueva oleada digital, y sean capaces de aumentar su competitividad y su capacidad de generación de nuevo empleo y con mayor calidad, son importantes. Sin embargo, los riesgos de pérdida de puestos de trabajo, exclusión de ocupaciones, caídas de la calidad del empleo y aumentos de la desigualdad también son evidentes si la balanza de la utilización de la robótica y la nueva oleada digital se decanta hacia la sustitución del trabajo. Una nueva orientación estratégica de los recursos humanos hacia la incorporación de la nueva oleada tecnológica, y nuevos sistemas y prácticas de gestión para capacitar y desarrollar a los empleados en el contexto de la co-robótica, parecen inevitables. 


\section{Referencias bibliográficas}

ACEMOGLOU, D.; RESTREPO, P. (2015). The race between man and machine: Implications of technology for growth, factor shares and employment. Paper no publicado. Cambridge, MA: MIT Press.

AUTOR, D. H. (2015). "Why are there still so many jobs? The history and future of workplace automation». Journal of Economic Perspectives. Vol. 29, núm. 3, págs. 3-30.

BESSEN, J. (2016). "Computers don't kill jobs but do increase inequality». Harvard Business Review (marzo).

BRYNJOLFSSON, E.; McAFEE, A. (2012). Race against the machine: How the digital revolution is accelerating innovation, driving productivity, and irreversibly transforming employment and the economy. Cambridge, MA: MIT Press.

BRYNJOLFSSON, E.; McAFEE, A. (2014). The second machine age: work, progress, and prosperity in a time of brilliant technologies. Nueva York: W. W. Norton \& Company.

FORD, M. (2015). Rise of the robots. Technology and the threat of a jobless future. Nueva York: Basic Books.

FREY, C. B.; OSBORNE, M. (2013). The future of employment: How susceptible are jobs to computerisation? Oxford: Oxford Martin School.

INTERNATIONAL FEDERATION OF ROBOTICS (2017). The impact of robots on productivity, employment and jobs. A positioning paper by the International Federation of Robotics. Frankfurt am Main: IFR.

PRATT, G. A. (2015). «Is a Cambrian explosion coming for robotics?». Journal of Economic Perspectives. Vol. 29, núm. 3, págs. 51-60.

ROTMAN, D. (2013). «How technology is destroying jobs». MIT Technology Review. Vol. 116, núm. 4, pags. 27-35.

TORRENT-SELLENS, J. (2015). "Knowledge products and network externalities. Implications for the business strategy». Journal of the Knowledge Economy. Vol. 6, núm. 1, págs. 138-156.

TORRENT-SELLENS, J.; FICAPAL-CUSÍ, P. (2009). TIC, conocimiento, redes y trabajo. Barcelona: Ediciones de la UOC. 


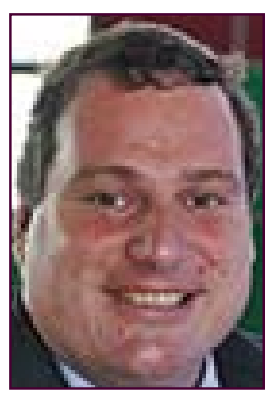

\section{Joan Torrent-Sellens jtorrent@uoc.edu Profesor de los Estudios de Economía y Empresa (UOC)}

Licenciado en Ciencias Económicas y Empresariales (UAB), máster en Análisis de Economía Aplicada (UAB) y doctor en Economía (Programa Sociedad Información y el Conocimiento) por la UOC. Director del grupo de investigación interdisciplinaria sobre las TIC, i2TIC (http://i2TIC. net) y profesor agregado (ámbito de economía) en los Estudios de Economía y Empresa de la UOC. Especialista en el análisis de las tecnologías de la información y la comunicación (TIC), la economía del conocimiento, y la empresa y el trabajo en red, temática sobre la que ha publicado veinticinco libros y setneta y cinco artículos en revistas de investigación indexadas.

Los textos publicados en esta revista están sujetas -salvo que se indique el contrario- a una licencia de Reconocimiento 3.0 España de Creative Commons. Podéis copiarlos, distribuirlos, comunicarlos públicamente y hacer obras derivadas siempre que reconozcáis los créditos de las obras (autoría, nombre de la revista, institución editora) de la manera especificada por los autores o por la revista. La licencia completa se puede consultar en http://creativecommons.org/licenses/by/3.0/es/deed.ca.

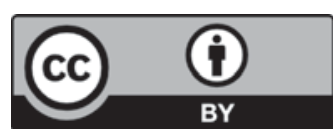

